https://doi.org/10.15407/ujpe65.5.385

M. MOLLA GESSESSE

Department of Physics, Aksum University

(P. O. Box 1010, Axum, Ethiopia; e-mail: mokemolla@gmail.com)

\title{
THE NOISE EFFECT OF VACUUM RESERVOIR ON THE DYNAMICS OF THREE-LEVEL LASER PUMPED BY COHERENT LIGHT
}

\begin{abstract}
We have investigated the effects of vacuum reservoir noise on the photon statistics and quadrature squeezing of light generated by a three-level laser pumped by coherent light and coupled to a vacuum reservoir via a single-port mirror. We have found that the effect of the vacuum reservoir noise is to increase the photon number variance and to decrease the quadrature squeezing. However, the vacuum reservoir noise has no effect on the mean photon number.

Keywords: stimulated emission, photon statistics, quadrature squeezing, Langevin noise operator.
\end{abstract}

\section{Introduction}

Squeezing is one of the interesting nonclassical features of light that has attracted the attention of many authors [1-11]. In a squeezed light, the quantum noise in one quadrature is below the vacuum level at the expense of enhanced fluctuations in the conjugate quadrature, such that the uncertainty relation is not violated. Squeezed light has potential applications in low-noise optical communications [12] and the weak signal detection [13-15].

Over the years, two types of a three-level laser have been studied by several authors. In type-one threelevel lasers, three-level atoms initially prepared in a coherent superposition of the top and bottom levels are injected at a constant rate into a cavity and then removed after they have spontaneously decayed to a level below the bottom level. In type-two three-level lasers, the top and bottom levels of the three-level atoms injected into a laser cavity are coupled by a strong coherent light.

Some authors investigated the statistical and squeezing properties of the light produced by typeone three-level laser [3-7]. N. Lu et al. [6] analyzed such properties of the light generated by a degenerate type-one three-level laser and found that the cavity laser light is in a squeezed state, when there are more atoms initially in the bottom levels than in the top level. Moreover, Lu and Zhu [3] analyzed a nondegenerate type-one three-level laser. They predicted a

(C) M. MOLLA GESSESSE, 2020

ISSN 2071-0194. Ukr. J. Phys. 2020. Vol. 65, No. 5 intracavity two-mode squeezing equal maximally to $50 \%$. It was found that the coherent superposition of the top and the bottom levels is responsible for the squeezing of light generated by such three-level lasers.

The statistical and squeezing properties of the light generated by type-two three-level laser were studied in [8]. Ansari et al. [8] considered a degenerate type-two three-level laser and predicted that the system generates a squeezed light under certain conditions. Furthermore, Ansari [4] studied a nondegenerate type-two three-level laser and found that the coupling of the top and bottom levels are responsible for the squeezing of the light generated by these types of three-level lasers.

In addition, some authors studied the statistical and squeezing properties of the light produced by three-level lasers in which the injected atoms are initially prepared in a coherent superposition of the top and bottom levels, and these levels are coupled after the injection into the cavity [4].

Preparing the three-level atoms in a coherent superposition of the top and bottom levels before they are injected into a laser cavity is not a simple task. Moreover, it may not possible to know whether the atoms have decayed spontaneously to levels other than the intermediate or bottom level before they are removed from the laser cavity [6]. In addition, when the top and bottom levels of the three-level lasers are coupled by strong coherent light, the atoms may decay from the top to bottom levels. This de- 
cay may decrease the mean photon number in the cavity.

Recently, Fesseha [2] studied a three-level laser in which three-level atoms available in a closed cavity are pumped from the bottom to the top level by the electron bombardment. He carried out his analysis by putting the vacuum noise operators in the normal order. It is not hard to realize that the normally ordered noise operators will not have any effect on the photon statistics and quadrature squeezing. He found that the light generated by the three-level laser operating far below the threshold is in a squeezed state, with the maximum quadrature squeezing being $50 \%$ below the vacuum-state level.

Furthermore, Fesseha [2] studied a three-level laser in which three-level atoms available in a closed cavity are pumped from the bottom to the top level by coherent light. He carried out his analysis by putting again the vacuum noise operators in the normal order. He found that the light generated by a threelevel laser is in a squeezed state, with the maximum quadrature squeezing being about $43 \%$ below the vacuum-state level.

Here, we wish to study the effects of the vacuum reservoir noise on the photon statistics and quadrature squeezing of the light generated by three-level atoms available in a closed cavity and pumped to the top level by coherent light. To this end, we firstly determine the quantum Langevin equations for the cavity mode operators and the equations of evolution of the atomic operators. Moreover, we obtain the solutions of the resulting equations. Applying these solutions, we calculate the photon statistics and quadrature squeezing of the two-mode cavity light and then discuss the effects of the vacuum reservoir noise.

\section{Equations of Evolution for Atomic and Cavity Mode Operators}

We consider here the case where $N$ three-level atoms, in a cascade configuration, are available in a closed cavity. We represent the top, intermediate, and bottom levels of the $k^{\text {th }}$ three-level atom by $\left|a_{k}\right\rangle,\left|b_{k}\right\rangle$, and $\left|c_{k}\right\rangle$, respectively. We assume the transitions $\left|a_{k}\right\rangle \rightarrow\left|b_{k}\right\rangle$ and $\left|b_{k}\right\rangle \rightarrow\left|c_{k}\right\rangle$ to be dipole allowed, with direct transitions $\left|a_{k}\right\rangle \rightarrow\left|c_{k}\right\rangle$ to be dipole forbidden. In addition, we consider the case where the the cavity modes are at resonance with the two transitions $\left|a_{k}\right\rangle \rightarrow\left|b_{k}\right\rangle$ and $\left|b_{k}\right\rangle \rightarrow\left|c_{k}\right\rangle$. When a three-level atom makes a transition from the top level to the intermediate level, it emits a photon with frequency $\omega_{a}$. It then undergoes a transition from the intermediate level to the bottom level by emitting a photon with frequency $\omega_{b}$.

The interaction of one of the three-level atoms with the two-mode cavity light and the pump mode can be described by the Hamiltonian [2]

$\hat{H}=i g\left[\hat{\sigma}_{a}^{\dagger k} \hat{a}-\hat{a}^{\dagger} \hat{\sigma}_{a}^{k}+\hat{\sigma}_{b}^{\dagger k} \hat{b}-\hat{b}^{\dagger} \hat{\sigma}_{b}^{k}\right]+\frac{i \Omega}{2}\left[\hat{\sigma}_{c}^{\dagger k}-\hat{\sigma}_{c}^{k}\right]$

where $g$ is the coupling constant between the threelevel atom and light mode $a$ or $b, \hat{a}(\hat{b})$ is the annihilation operator for the two-mode cavity light, and $\Omega$ is a constant proportional to the amplitude of the coherent light. Here, the lowering atomic operators are defined by $\hat{\sigma}_{a}^{k}=\left|b_{k}\right\rangle\left\langle a_{k}\left|, \hat{\sigma}_{b}^{k}=\right| c_{k}\right\rangle\left\langle b_{k}\right|$, and $\hat{\sigma}_{c}^{k}=$ $=\left|c_{k}\right\rangle\left\langle a_{k}\right|$.

Applying Eq. (1), the quantum Langevin equations for the cavity mode operators $\hat{a}$ and $\hat{b}$ are found to be

$$
\begin{aligned}
& \frac{d \hat{a}}{d t}=-\frac{\kappa}{2} \hat{a}-g \hat{\sigma}_{a}^{k}+\hat{F}_{a}(t), \\
& \frac{d \hat{b}}{d t}=-\frac{\kappa}{2} \hat{b}-g \hat{\sigma}_{b}^{k}+\hat{F}_{b}(t),
\end{aligned}
$$

where $\kappa$ is the cavity damping constant, and $\hat{F}_{a}\left(\hat{F}_{b}\right)$ is a vacuum reservoir noise operator associated with a cavity mode operator. Furthermore, using the relation

$\frac{d}{d t}\langle\hat{A}\rangle=-i\langle[\hat{A}, \hat{H}]\rangle$

along with the interaction Hamiltonian given by (1), we readily find

$$
\begin{aligned}
& \frac{d}{d t}\left\langle\hat{\sigma}_{a}^{k}\right\rangle=g\left\langle\hat{\eta}_{b}^{k} \hat{a}\right\rangle-g\left\langle\hat{\eta}_{a}^{k} \hat{a}\right\rangle+g\left\langle\hat{b}^{\dagger} \hat{\sigma}_{c}^{k}\right\rangle+\frac{\Omega}{2}\left\langle\hat{\sigma}_{b}^{\dagger k}\right\rangle \\
& \frac{d}{d t}\left\langle\hat{\sigma}_{b}^{k}\right\rangle=g\left\langle\hat{\eta}_{c}^{k} \hat{b}\right\rangle-g\left\langle\hat{\eta}_{b}^{k} \hat{b}\right\rangle-g\left\langle\hat{a}^{\dagger} \hat{\sigma}_{c}^{k}\right\rangle-\frac{\Omega}{2}\left\langle\hat{\sigma}_{a}^{\dagger k}\right\rangle \\
& \frac{d}{d t}\left\langle\hat{\sigma}_{c}^{k}\right\rangle=g\left\langle\hat{\sigma}_{b}^{k} \hat{a}\right\rangle-g\left\langle\hat{\sigma}_{a}^{k} \hat{b}\right\rangle+\frac{\Omega}{2}\left[\left\langle\hat{\eta}_{c}^{k}\right\rangle-\left\langle\hat{\eta}_{a}^{k}\right\rangle\right] \\
& \frac{d}{d t}\left\langle\hat{\eta}_{a}^{k}\right\rangle=g\left\langle\hat{\sigma}_{a}^{\dagger k} \hat{a}\right\rangle+g\left\langle\hat{a}^{\dagger} \hat{\sigma}_{a}^{k}\right\rangle+\frac{\Omega}{2}\left[\left\langle\hat{\sigma}_{c}^{\dagger k}\right\rangle+\left\langle\hat{\sigma}_{c}^{k}\right\rangle\right] \\
& \frac{d}{d t}\left\langle\hat{\eta}_{b}^{k}\right\rangle=g\left\langle\hat{\sigma}_{b}^{\dagger k} \hat{b}\right\rangle-g\left\langle\hat{\sigma}_{a}^{\dagger k} \hat{a}\right\rangle-g\left\langle\hat{a}^{\dagger} \hat{\sigma}_{a}^{k}\right\rangle+g\left\langle\hat{b}^{\dagger} \hat{\sigma}_{b}^{k}\right\rangle \\
& \frac{d}{d t}\left\langle\hat{\eta}_{c}^{k}\right\rangle=-g\left\langle\hat{\sigma}_{b}^{\dagger k} \hat{b}\right\rangle-g\left\langle\hat{b}^{\dagger} \hat{\sigma}_{b}^{k}\right\rangle-\frac{\Omega}{2}\left[\left\langle\hat{\sigma}_{c}^{\dagger k}\right\rangle+\left\langle\hat{\sigma}_{c}^{k}\right\rangle\right]
\end{aligned}
$$


where $\hat{\eta}_{a}^{k}=\left|a_{k}\right\rangle\left\langle a_{k}\left|, \hat{\eta}_{b}^{k}=\right| b_{k}\right\rangle\left\langle b_{k}\left|, \hat{\eta}_{c}^{k}=\right| c_{k}\right\rangle\left\langle c_{k}\right|$. We see that Eqs. (5)-(10) are nonlinear coupled differential equations, and it is not possible to find the exact time-dependent solutions of these equations. We intend to overcome this problem by applying the largetime approximation scheme [11]. Then, in this approximation scheme, we get the following approximately valid relations from (2) and (3):

$\hat{a}=-\frac{2 g}{\kappa} \hat{\sigma}_{a}^{k}+\frac{2}{\kappa} \hat{F}_{a}(t)$,
$\hat{b}=-\frac{2 g}{\kappa} \hat{\sigma}_{b}^{k}+\frac{2}{\kappa} \hat{F}_{b}(t)$.

Substituting Eqs. (11) and (12) into (5)-(10), we arrive at

$\frac{d}{d t}\left\langle\hat{\sigma}_{a}^{k}\right\rangle=-\gamma_{c}\left\langle\hat{\sigma}_{a}^{k}\right\rangle+\frac{2 g}{\kappa}\left[\left\langle\hat{\eta}_{b}^{k} \hat{F}_{a}-\hat{\eta}_{a}^{k} \hat{F}_{a}+\hat{F}_{b}^{\dagger} \hat{\sigma}_{c}^{k}\right\rangle\right]+$

$+\frac{\Omega}{2}\left\langle\hat{\sigma}_{b}^{\dagger k}\right\rangle$

$\frac{d}{d t}\left\langle\hat{\sigma}_{b}^{k}\right\rangle=-\frac{\gamma_{c}}{2}\left\langle\hat{\sigma}_{b}^{k}\right\rangle+\frac{2 g}{\kappa}\left[\left\langle\hat{\eta}_{c}^{k} \hat{F}_{b}-\hat{\eta}_{a}^{k} \hat{F}_{b}-\hat{F}_{a}^{\dagger} \hat{\sigma}_{c}^{k}\right\rangle\right]-$

$-\frac{\Omega}{2}\left\langle\hat{\sigma}_{a}^{\dagger k}\right\rangle$

$\frac{d}{d t}\left\langle\hat{\sigma}_{c}^{k}\right\rangle=-\frac{\gamma_{c}}{2}\left\langle\hat{\sigma}_{c}^{k}\right\rangle+\frac{2 g}{\kappa}\left[\left\langle\hat{\sigma}_{b}^{k} \hat{F}_{a}-\hat{\sigma}_{a}^{k} \hat{F}_{b}\right\rangle\right]+$

$+\frac{\Omega}{2}\left[\left\langle\hat{\eta}_{c}^{k}-\hat{\eta}_{a}^{k}\right\rangle\right]$

$\frac{d}{d t}\left\langle\hat{\eta}_{a}^{k}\right\rangle=-\gamma_{c}\left\langle\hat{\eta}_{a}^{k}\right\rangle+\frac{2 g}{\kappa}\left[\left\langle\hat{\sigma}_{a}^{\dagger k} \hat{F}_{a}+\hat{F}_{a}^{\dagger} \hat{\sigma}_{a}^{k}\right\rangle\right]+$

$+\frac{\Omega}{2}\left[\left\langle\hat{\sigma}_{c}^{\dagger k}+\hat{\sigma}_{c}^{k}\right\rangle\right]$

$\frac{d}{d t}\left\langle\eta_{b}^{k}\right\rangle=\gamma_{c}\left[\left\langle\hat{\eta}_{a}^{k}-\hat{\eta}_{b}^{k}\right\rangle\right]+$

$+\frac{2 g}{\kappa}\left[\left\langle\hat{\sigma}_{b}^{\dagger k} \hat{F}_{b}-\hat{\sigma}_{a}^{\dagger k} \hat{F}_{a}+\hat{F}_{b}^{\dagger} \hat{\sigma}_{b}^{k}-\hat{F}_{a}^{\dagger} \hat{\sigma}_{a}^{k}\right\rangle\right]$,

$\frac{d}{d t}\left\langle\hat{\eta}_{c}^{k}\right\rangle=\gamma_{c}\left\langle\hat{\eta}_{b}^{k}\right\rangle-\frac{2 g}{\kappa}\left[\left\langle\hat{\sigma}_{b}^{\dagger k} \hat{F}_{a}+\hat{F}_{b}^{\dagger} \hat{\sigma}_{b}^{k}\right\rangle\right]-$

$-\frac{\Omega}{2}\left[\left\langle\hat{\sigma}_{c}^{\dagger k}+\hat{\sigma}_{c}^{k}\right\rangle\right]$

where $\gamma_{c}=4 g^{2} / \kappa$ is the stimulated emission decay constant [2].

Next, we wish to determine the values of the terms inside the square brackets indicated in (13). To this end, after removing the angular brackets, the formal solution of Eq. (16) can be written as

$\hat{\eta}_{a}^{k}(t)=\hat{\eta}_{a}^{k}(0) e^{-\gamma_{c} t}+$

ISSN 2071-0194. Ukr. J. Phys. 2020. Vol. 65, No. 5 $+\frac{2 g}{\kappa} \int_{0}^{t} d t^{\prime} e^{-\gamma_{c}\left(t-t^{\prime}\right)}\left[\hat{\sigma}_{a}^{\dagger k}\left(t^{\prime}\right) \hat{F}_{a}\left(t^{\prime}\right)+\hat{F}_{a}^{\dagger}\left(t^{\prime}\right) \hat{\sigma}_{a}^{k}\left(t^{\prime}\right)\right]+$

$+\int_{0}^{t} d t^{\prime} e^{-\gamma_{c}\left(t-t^{\prime}\right)}\left[\frac{\Omega}{2}\left(\hat{\sigma}_{c}^{\dagger k}\left(t^{\prime}\right)+\hat{\sigma}_{c}^{k}\left(t^{\prime}\right)\right)+\hat{G}\left(t^{\prime}\right)\right]$,

where $\hat{G}(t)$ is a vacuum reservoir noise operator associated with an atomic operator. Multiplying the above equation from the right by $\hat{F}_{a}(t)$ and taking the expectation value of the resulting expression, we get

$\left\langle\hat{\eta}_{a}^{k}(t) \hat{F}_{a}(t)\right\rangle=\left\langle\hat{\eta}_{a}^{k}(0) \hat{F}_{a}(t)\right\rangle e^{-\gamma_{c} t}+$

$+\int_{0}^{t} d t^{\prime} e^{-\gamma_{c}\left(t-t^{\prime}\right)}\left[\left\{\left\langle\hat{\sigma}_{a}^{\dagger k}\left(t^{\prime}\right) \hat{F}_{a}\left(t^{\prime}\right) \hat{F}_{a}(t)\right\rangle+\right.\right.$

$\left.+\left\langle\hat{F}_{a}^{\dagger}\left(t^{\prime}\right) \hat{\sigma}_{a}^{k}\left(t^{\prime}\right) \hat{F}_{a}(t)\right\rangle\right\} \frac{2 g}{\kappa}+\frac{\Omega}{2}\left\{\left\langle\hat{\sigma}_{c}^{\dagger k}\left(t^{\prime}\right) \hat{F}_{a}(t)\right\rangle+\right.$

$\left.\left.+\left\langle\hat{\sigma}_{c}^{k}\left(t^{\prime}\right) \hat{F}_{a}(t)\right\rangle\right\}+\left\langle\hat{G}\left(t^{\prime}\right) \hat{F}_{a}(t)\right\rangle\right]$.

Considering the fact that the noise operator $\hat{F}_{a}(t)$ at a certain time does not affect the atomic operator at earlier time and assuming that there is no correlation between the atomic and vacuum noise operators, Eq. (20) can be put in the form

$$
\begin{aligned}
& \left\langle\hat{\eta}_{a}^{k}(t) \hat{F}_{a}(t)\right\rangle=\frac{2 g}{\kappa} \int_{0}^{t} d t^{\prime} e^{-\gamma_{c}\left(t-t^{\prime}\right)} \times \\
& \times\left[\left\langle\hat{\sigma}_{a}^{\dagger k}\left(t^{\prime}\right) \hat{F}_{a}\left(t^{\prime}\right) \hat{F}_{a}(t)\right\rangle+\left\langle\hat{F}_{a}^{\dagger}\left(t^{\prime}\right) \hat{\sigma}_{a}^{k}\left(t^{\prime}\right) \hat{F}_{a}(t)\right\rangle\right] .
\end{aligned}
$$

It is impossible to evaluate the integral that appears in Eq. (21), as the explicit form of $\hat{\sigma}_{a}^{k}\left(t^{\prime}\right)$ is unknown yet. In order to proceed further, we need to adopt a certain approximation scheme. To this end, ignoring the noncommutativity of the atomic and noise operators [9], we see that

$$
\left\langle\hat{F}_{a}^{\dagger}\left(t^{\prime}\right) \hat{\sigma}_{a}^{k}\left(t^{\prime}\right) \hat{F}_{a}(t)\right\rangle=\left\langle\hat{\sigma}_{a}^{k}\left(t^{\prime}\right) \hat{F}_{a}^{\dagger}\left(t^{\prime}\right) \hat{F}_{a}(t)\right\rangle
$$

Neglecting now the correlation between $\hat{\sigma}_{a}^{k}\left(t^{\prime}\right)$ and $\hat{F}_{a}^{\dagger}\left(t^{\prime}\right) \hat{F}_{a}(t)$, we can write the approximately valid relation

$\left\langle\hat{\sigma}_{a}^{k}\left(t^{\prime}\right) \hat{F}_{a}^{\dagger}\left(t^{\prime}\right) \hat{F}_{a}(t)\right\rangle=\left\langle\hat{\sigma}_{a}^{k}\left(t^{\prime}\right)\right\rangle\left\langle\hat{F}_{a}^{\dagger}\left(t^{\prime}\right) \hat{F}_{a}(t)\right\rangle$. 
On the basis of the correlation properties of the noise operators found in Ref. [6], the above equation takes the form

$\left\langle\hat{\sigma}_{a}^{k}\left(t^{\prime}\right) \hat{F}_{a}^{\dagger}\left(t^{\prime}\right) \hat{F}_{a}(t)\right\rangle=0$.

The similar relation holds for $\left\langle\hat{\sigma}_{a}^{\dagger k}\left(t^{\prime}\right) \hat{F}_{a}\left(t^{\prime}\right) \hat{F}_{a}(t)\right\rangle$. Hence, in view of the above results, Eq. (21) can be put in the form

$\left\langle\hat{\eta}_{a}^{k}(t) \hat{F}_{a}(t)\right\rangle=0$.

Following a similar line of reasoning, we can also easily verify that

$\left\langle\hat{\eta}_{b}^{k}(t) \hat{F}_{a}(t)\right\rangle=0$

and

$\left\langle\hat{F}_{b}^{\dagger k}(t) \hat{\sigma}_{c}^{k}(t)\right\rangle=0$.

Substituting (25), (26), and (27) into Eq. (13), we get

$\frac{d}{d t}\left\langle\hat{\sigma}_{a}^{k}\right\rangle=-\gamma_{c}\left\langle\hat{\sigma}_{a}^{k}\right\rangle+\frac{\Omega}{2}\left\langle\hat{\sigma}_{b}^{\dagger k}\right\rangle$.

Now summing the resulting expression over all $N$ three-level atoms and making use of the mean field theory, we obtain

$\frac{d}{d t}\left\langle\hat{m}_{a}\right\rangle=-\gamma_{c}\left\langle\hat{m}_{a}\right\rangle+\frac{\Omega}{2}\left\langle\hat{m}_{b}^{\dagger}\right\rangle$.

Similarly, we can easily establish that

$$
\begin{aligned}
\frac{d}{d t}\left\langle\hat{m}_{b}\right\rangle & =-\frac{\gamma_{c}}{2}\left\langle\hat{m}_{b}\right\rangle-\frac{\Omega}{2}\left\langle\hat{m}_{a}^{\dagger}\right\rangle, \\
\frac{d}{d t}\left\langle\hat{m}_{c}\right\rangle & =-\frac{\gamma_{c}}{2}\left\langle\hat{m}_{c}\right\rangle+\frac{\Omega}{2}\left[\left\langle\hat{N}_{c}\right\rangle-\left\langle\hat{N}_{a}\right\rangle\right], \\
\frac{d}{d t}\left\langle\hat{N}_{a}\right\rangle & =-\gamma_{c}\left\langle\hat{N}_{a}\right\rangle+\frac{\Omega}{2}\left[\left\langle\hat{m}_{c}^{\dagger}\right\rangle+\left\langle\hat{m}_{c}\right\rangle\right], \\
\frac{d}{d t}\left\langle\hat{N}_{b}\right\rangle & =\gamma_{c}\left\langle\hat{N}_{a}\right\rangle-\gamma_{c}\left\langle\hat{N}_{b}\right\rangle, \\
\frac{d}{d t}\left\langle\hat{N}_{c}\right\rangle & =\gamma_{c}\left\langle\hat{N}_{b}\right\rangle-\frac{\Omega}{2}\left[\left\langle\hat{m}_{c}^{\dagger}\right\rangle+\left\langle\hat{m}_{c}\right\rangle\right],
\end{aligned}
$$

in which

$\hat{m}_{a}=\sum \hat{\sigma}_{a}^{k}, \quad \hat{m}_{b}=\sum \hat{\sigma}_{b}^{k}, \quad$ and $\quad \hat{m}_{c}=\sum \hat{\sigma}_{c}^{k}$,

$\hat{N}_{a}=\sum \hat{\eta}_{a}^{k}, \quad \hat{N}_{b}=\sum \hat{\eta}_{b}^{k}, \quad$ and $\quad \hat{N}_{c}=\sum \hat{\eta}_{c}^{k}$ where the operators $\hat{N}_{a}, \hat{N}_{b}$, and $\hat{N}_{c}$ represent the number of atoms on the upper, intermediate, and bottom levels.

We note that the steady-state solutions of Eqs. (31), (32), and (33) are

$$
\begin{aligned}
& \left\langle\hat{m}_{c}\right\rangle=\frac{\Omega}{\gamma_{c}}\left[\left\langle\hat{N}_{c}\right\rangle-\left\langle\hat{N}_{a}\right\rangle\right], \\
& \left\langle\hat{N}_{a}\right\rangle=\frac{\Omega}{2 \gamma_{c}}\left[\left\langle\hat{m}_{c}^{\dagger}\right\rangle+\left\langle\hat{m}_{c}\right\rangle\right], \\
& \left\langle\hat{N}_{a}\right\rangle=\left\langle\hat{N}_{b}\right\rangle .
\end{aligned}
$$

From whence, we have

$$
\begin{aligned}
& \left\langle\hat{N}_{a}\right\rangle=\left[\frac{\Omega^{2}}{3 \Omega^{2}+\gamma_{c}^{2}}\right] N=\left[\frac{\eta^{2}}{1+3 \eta^{2}}\right] N, \\
& \left\langle\hat{N}_{c}\right\rangle=\left[\frac{\Omega^{2}+\gamma_{c}^{2}}{3 \Omega^{2}+\gamma_{c}^{2}}\right] N=\left[\frac{1+\eta^{2}}{1+3 \eta^{2}}\right] N, \\
& \left\langle\hat{m}_{c}\right\rangle=\left[\frac{\Omega \gamma_{c}}{3 \Omega^{2}+\gamma_{c}^{2}}\right] N=\left[\frac{\eta}{1+3 \eta^{2}}\right] N,
\end{aligned}
$$

where $\Omega / \gamma_{c}=\eta$. On the other hand, when the cavity modes are interacting with all three-level atoms available in the cavity, we can rewrite expression (2) in the form [2]

$\frac{d \hat{a}}{d t}=-\frac{\kappa}{2} \hat{a}+\alpha \hat{m}_{a}+\beta \hat{F}_{a}(t)$

where $\alpha$ and $\beta$ are constants whose values remain to be fixed. Applying the steady-state solution of (2) and summing the resulting expression over all atoms, we obtain

$\left[\hat{a}, \hat{a}^{\dagger}\right]=\frac{\gamma_{c}}{\kappa}\left[\hat{N}_{b}-\hat{N}_{a}\right]+\frac{4 N}{\kappa^{2}}\left[\hat{F}_{a}, \hat{F}_{a}^{\dagger}\right]$.

Moreover, using the steady-state solution of (43), we easily arrive at

$\left[\hat{a}, \hat{a}^{\dagger}\right]=N\left[\frac{2 \alpha}{\kappa}\right]^{2}\left[\hat{N}_{b}-\hat{N}_{a}\right]+\frac{4 \beta^{2}}{\kappa^{2}}\left[\hat{F}_{a}, \hat{F}_{a}^{\dagger}\right]$.

Thus, the comparison of Eq. (44) with (45) indicates that

$\alpha= \pm \frac{g}{\sqrt{N}} \quad$ and $\quad \beta= \pm \sqrt{N}$.

Now employing Eq. (46) in (43), the equation of evolution for the cavity mode operator $\hat{a}$ can be rewritten as

$\frac{d \hat{a}}{d t}=-\frac{\kappa}{2} \hat{a}+\frac{g}{\sqrt{N}} \hat{m}_{a}+\sqrt{N} \hat{F}_{a}(t)$.

ISSN 2071-0194. Ukr. J. Phys. 2020. Vol. 65, No. 5 
Similarly, we find the equation of evolution for the cavity mode operator $\hat{b}$ to be

$\frac{d \hat{b}}{d t}=-\frac{\kappa}{2} \hat{b}+\frac{g}{\sqrt{N}} \hat{m}_{b}+\sqrt{N} \hat{F}_{b}(t)$.

Furthermore, adding Eq. (47) and (48), we get

$\frac{d \hat{c}}{d t}=-\frac{\kappa}{2} \hat{c}+\frac{g}{\sqrt{N}} \hat{m}+\sqrt{N} \hat{F}_{c}(t)$,

where $\hat{c}=\hat{a}+\hat{b}, \hat{m}=\hat{m}_{a}+\hat{m}_{b}$, and $\hat{F}_{c}=\hat{F}_{a}+$ $+\hat{F}_{b}$. Now using $\left\langle\hat{F}_{c}\right\rangle=0$ along with the assumptions that the three-level atoms are initially in the ground level and the cavity modes are initially in a vacuum state, we find the solution of the expectation value of the operator $\hat{c}$ to be zero. Hence, on the basis of this result and (49), we can conclude that $\hat{c}$ is a Gaussian variable with zero mean.

\section{Photon Statistics}

Here, we will obtain the mean photon number and the photon-number variance for a two-mode light beam. Employing the relation

$\frac{d}{d t}\left\langle\hat{c}^{\dagger} \hat{c}\right\rangle=\left\langle\frac{d \hat{c}^{\dagger}}{d t} \hat{c}\right\rangle+\left\langle\hat{c}^{\dagger} \frac{d \hat{c}}{d t}\right\rangle$

along with the equation of evolution given by (49) and its adjoint, we obtain

$\frac{d}{d t}\left\langle\hat{c}^{\dagger} \hat{c}\right\rangle=-\kappa\left\langle\hat{c}^{\dagger} \hat{c}\right\rangle+\frac{\gamma_{c}}{N}\left\langle\hat{m}^{\dagger} \hat{m}\right\rangle$

The steady-state solution of Eq. (51) is found, by taking into account that $\left\langle\hat{m}^{\dagger} \hat{m}\right\rangle=N\left(\left\langle\hat{N}_{a}\right\rangle+\left\langle\hat{N}_{b}\right\rangle\right)$, to be

$\left\langle\hat{c}^{\dagger} \hat{c}\right\rangle=\frac{\gamma_{c}}{\kappa}\left[\left\langle\hat{N}_{a}\right\rangle+\left\langle\hat{N}_{b}\right\rangle\right]$

Therefore, in view of Eqs. (40) and (41), the above equation takes the form

$\left\langle\hat{c}^{\dagger} \hat{c}\right\rangle=\frac{\gamma_{c}}{\kappa}\left[\frac{2 \eta^{2}}{1+3 \eta^{2}}\right] N$

In order to investigate the dependence of the mean photon number on the amplitude of the driving coherent light, number of atoms available in the cavity, stimulated emission decay constant, and cavity damping constant, we can plot the mean photon number

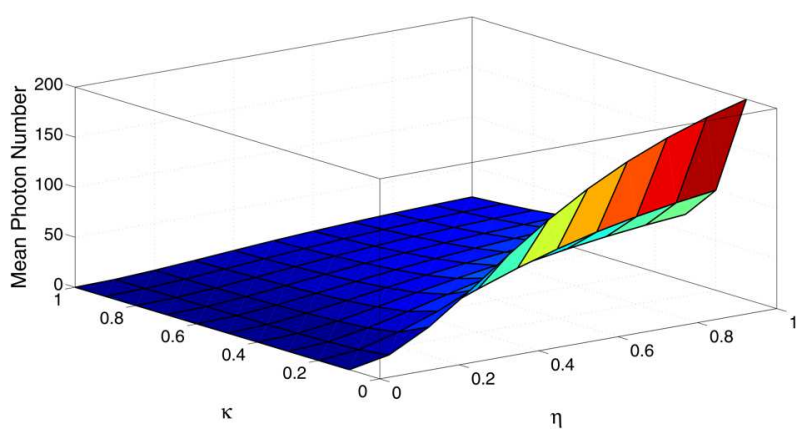

Fig. 1. Plot of $\left\langle\hat{c}^{\dagger} \hat{c}\right\rangle$ [Eq. (53)] versus $\eta$ and $\kappa$ for $\gamma_{c}=0.4$ and $N=100$

versus these parameters. To this end, we only consider the dependence of the mean photon number on the amplitude of the driving coherent light and on the cavity damping constant, with the number of atoms and stimulated emission decay constant being fixed. So, we only plot the mean photon number given by Eq. (53) versus $\eta$ and $\kappa$. In this case, the total number of atoms and stimulated emission decay constant are taken to be 100 and 0.4 respectively. For example, it is found from Fig. 1 that, when $\eta=0$ and for any values of $\kappa$, the mean number of photons is zero. However, for $\eta=1$ and $\kappa=0.1$, the mean number of photons emitted by the three-level atoms is found to be 200 . We can also assure you that there is no change in the mean photon number, whether the system of interest is damped by the vacuum reservoir or not.

We now obtain the steady-state photon-number variance of the two-mode cavity light defined by

$(\Delta n)^{2}=\left\langle\left(\hat{c}^{\dagger} \hat{c}\right)^{2}\right\rangle-\left\langle\hat{c}^{\dagger} \hat{c}\right\rangle^{2}$.

Since $\hat{c}$ is a Gaussian variable with zero mean, the photon-number variance of the two-mode cavity light can be put in the form [11]

$(\Delta n)^{2}=\left\langle\hat{c}^{\dagger} \hat{c}\right\rangle\left\langle\hat{c} \hat{c}^{\dagger}\right\rangle+\left\langle\hat{c}^{\dagger}\right\rangle\left\langle\hat{c}^{2}\right\rangle$.

We can readily establish that

$\left\langle\hat{c} \hat{c}^{\dagger}\right\rangle=\frac{\gamma_{c}}{\kappa}\left[\frac{1+2 \eta^{2}}{1+3 \eta^{2}}\right] N+2 N$

and

$\left\langle\hat{c}^{2}\right\rangle=\frac{\gamma_{c}}{\kappa}\left[\frac{\eta}{1+3 \eta^{2}}\right] N$

With the aid of (53), (56), and (57), the photonnumber variance of the two-mode cavity light given 


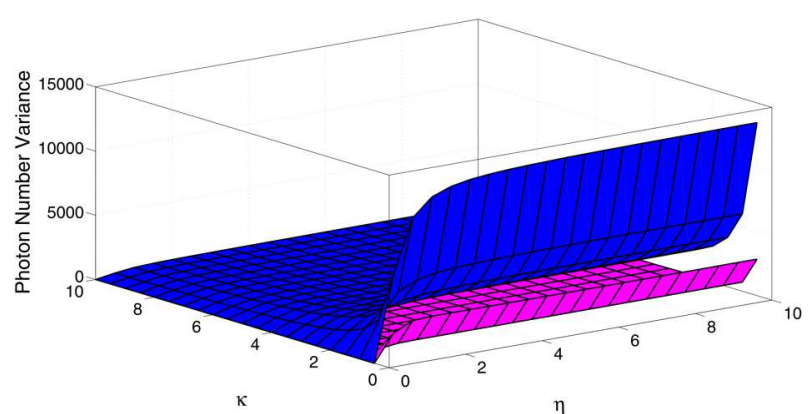

Fig. 2. Plots of the photon number variances [Eq. (58)] versus $\eta$ and $\kappa$ for $\gamma_{c}=0.4$ and $N=100$

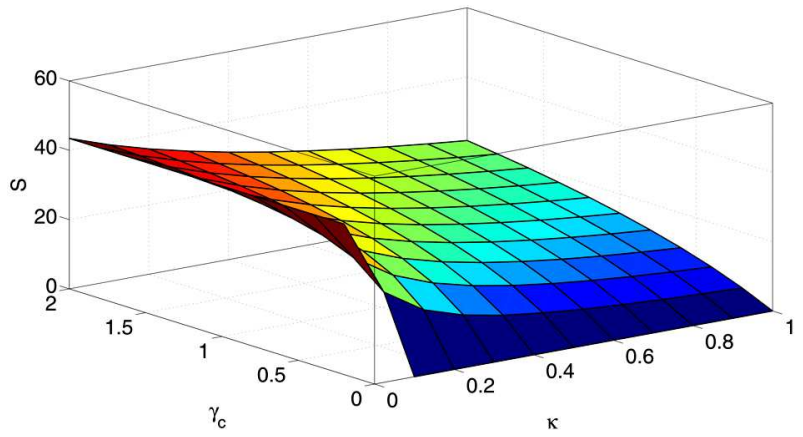

Fig. 3. Plots of the quadrature squeezing [Eq. (69)] versus $\kappa$ and $\gamma_{c}$

by (55) is found to be

$$
\begin{aligned}
& (\Delta n)^{2}=\left[\frac{\gamma_{c}}{\kappa}\right]^{2}\left[\left(\frac{2 N \eta^{2}}{1+3 \eta^{2}}\right)^{2}+3\left(\frac{N \eta}{1+3 \eta^{2}}\right)^{2}\right]+ \\
& +\frac{4 \gamma_{c}}{\kappa}\left[\frac{N^{2} \eta^{2}}{1+3 \eta^{2}}\right] .
\end{aligned}
$$

Now, we are in a position to plot the photon number variance with and without the last term on the right-hand side of the above expression. On comparing the plots having the magenta color, the two-mode cavity light is not damped by the two-mode vacuum reservoir, the one obtained in Ref. [2], and blue color, the two-mode cavity light is actually damped by the two-mode vacuum reservoir, we see that the effect of the vacuum reservoir noise is to increase the photonnumber variance by a significant amount, as already shown in Fig. 2. However, as we have seen previously, the vacuum reservoir noise does not have any effect on the mean photon number.

\section{Quadrature Squeezing}

The squeezing properties of the two-mode light are described by two quadrature operators defined by

$\hat{c}_{+}=\hat{c}^{\dagger}+\hat{c} \quad$ and $\quad \hat{c}_{-}=i\left(\hat{c}^{\dagger}-\hat{c}\right)$,

where $\hat{c}_{+}$and $\hat{c}_{-}$are Hermitian operators representing physical quantities, which we prefer to call the plus and minus quadratures. With the help of (59), we establish that the two quadrature operators satisfy the commutation relation

$\left[\hat{c}_{-}, \hat{c}_{+}\right]=2 i\left[\frac{\gamma_{c}}{\kappa}\left(\hat{N}_{a}-\hat{N}_{c}\right)-2 N\right]$.

It then follows that

$\Delta c_{+} \Delta c_{-} \geq\left|\frac{\gamma_{c}}{\kappa}\left(\left\langle\hat{N}_{a}\right\rangle-\left\langle\hat{N}_{c}\right\rangle\right)-2 N\right|$.

With the aid of Eqs. (40) and (41), the uncertainty relation for the two quadrature operators takes, at the steady-state, the form

$\Delta c_{+} \Delta c_{-} \geq \frac{\gamma_{c}}{\kappa}\left[\frac{N}{1+3 \eta^{2}}\right]+2 N$.

Furthermore, we consider the case where the coherent light does not couple the top and bottom levels. Thus, by setting $\eta=0$, the above equation reduces to

$\Delta c_{+} \Delta c_{-} \geq N\left[\frac{\gamma_{c}}{\kappa}+2\right]$.

We now proceed to calculate the quadrature variance of the two-mode cavity light. It is defined by

$\left(\Delta c_{ \pm}\right)^{2}=\left\langle\hat{c}_{ \pm}^{2}\right\rangle-\left\langle\hat{c}_{ \pm}\right\rangle^{2}$.

Accounting for (59) and using the fact that $\langle\hat{c}\rangle=0$, the quadrature variance can be put in the form

$\left(\Delta c_{ \pm}\right)^{2}=\left\langle\hat{c}^{\dagger} \hat{c}\right\rangle+\left\langle\hat{c} \hat{c}^{\dagger}\right\rangle \pm\left\langle\hat{c}^{2}\right\rangle \pm\left\langle\hat{c}^{\dagger 2}\right\rangle$

Substituting Eqs. (52), (56), and (57) into (65), the plus (minus) quadrature variance of the two-mode light is found, at the steady-state, to be

$\left(\Delta c_{ \pm}\right)^{2}=\frac{\gamma_{c}}{\kappa}\left[\frac{4 \eta^{2} \pm 2 \eta+1}{1+3 \eta^{2}}\right] N+2 N$.

We immediately notice that the two-mode cavity light is in a squeezed state, and the squeezing occurs in ISSN 2071-0194. Ukr. J. Phys. 2020. Vol. 65, No. 5 
the minus quadrature. Moreover, by setting $\eta=0$ in Eq. (66), we easily get

$\left(\Delta c_{ \pm}\right)_{v}^{2}=N\left[\frac{\gamma_{c}}{\kappa}+2\right]$

which is the quadrature variance of a two-mode vacuum state. We note that, for $\eta=0$, the uncertainty in the plus and minus quadratures are equal and satisfy the minimum uncertainty relation, as can be seen from Eqs. (63) and (67).

Finally, we determine the quadrature squeezing of the cavity light relative to the quadrature variance of the cavity vacuum sate defined by [2]:

$S=\frac{\left(\Delta c_{-}\right)_{v}^{2}-\left(\Delta c_{-}\right)^{2}}{\left(\Delta c_{-}\right)_{v}^{2}}$

By substituting the minus quadrature variances given by (66) and (67) into (68), the steady-state quadrature squeezing of the two-mode cavity light is found to be

$S=\left[\frac{\gamma_{c}}{\gamma_{c}+2 \kappa}\right]\left[\frac{2 \eta-\eta^{2}}{1+3 \eta^{2}}\right]$.

From Fig. 3, we clearly see that the two-mode cavity radiation exhibits no squeezing for certain values of $\kappa$ and $\gamma_{c}$ even when it is coupled to a two-mode vacuum reservoir. The second term on the right-hand side of the above expression is already obtained in Ref.[2], whose level of maximum quadrature squeezing with respect to the vacuum state is already found to be $43.43 \%$. As the cavity damping constant $\kappa$ is decreasing, and the stimulated emission decay constant $\gamma_{c}$ is increasing, the two-mode cavity radiation does exhibit a maximum quadrature squeezing which is exactly equal to the one obtained in Ref. [2].

Following the same line of reasoning, by comparing this result with the one obtained in Ref. [2], we see that the effect of the vacuum reservoir noise is to decrease the quadrature squeezing by a factor of

$\left[\frac{\gamma_{c}}{\gamma_{c}+2 \kappa}\right]$

\section{Conclusion}

We have studied the effects of the vacuum reservoir noise on the statistical and squeezing properties of the light modes produced by a three-level laser. In this optical system, $N$ three-level atoms available in a closed cavity coupled to a two-mode vacuum reservoir are pumped from the bottom to the top level by means of coherent light. Based on the definition given by $\gamma_{c}=4 g^{2} / \kappa$, we conclude that an atom in the top or middle level and inside a closed cavity emits a photon due to its interaction with the cavity light. We certainly identify this process to be a stimulated emission.

Employing the interaction Hamiltonian and the Heisenberg equation of motion, we have obtained the quantum Langevin equations for the cavity mode operators and the equations of evolution of the expectation values of the atomic operators. Making use of the formal (approximated) solutions of the quantum Langevin equations for the cavity mode operators and the formal (approximated) solutions of the equations of evolution of the expectation values of the atomic operators, we have calculated the mean photon number and the photon-number variance of the two-mode cavity light. The photon-number variance of the two-mode cavity light of this work is observed to be greater than that of the photon-number variance studied by Fesseha Ref. [2]. We have realized that this difference is due to the effect of the vacuum reservoir noise.

We have also calculated the quadrature squeezing of the two-mode cavity light produced by a three-level laser. It has been found that the three-level laser produces a squeezed light under a certain condition. The maximum quadrature squeezing is found to be about $31 \%$ below the vacuum-state level. This quadrature squeezing is observed to be less than that of a threelevel laser studied by Fesseha Ref. [2], in which the maximum quadrature squeezing is about $43 \%$ below the vacuum-state level. We have realized that this difference is due to the effect of the vacuum reservoir noise.

1. M.O. Scully, M.S. Zubairy. Quantum Optics (Cambridge Univ. Press, 1997).

2. Fesseha Kassahun. Refind Quantum Analysis of Light (Create Space Independent Publ. Platform, 2014).

3. M.O. Scully, M.S. Zubairy. Noise free amplification via the two-photon correlated spontaneous emission laser. Opt. Commun. 66, 303 (1988).

4. J. Anwar, M.S. Zubairy. Quantum-statistical properties of noise in a phase-sensitive linear amplifier. Phys. Rev. A 49, 481 (1994).

5. N.Lu, F.X. Zhao, J. Bergou. Nonlinear theory of a twophoton correlated-spontaneous-emission laser: A coher- 
ently pumped two-level-two-photon laser. Phys. Rev. A 39 , 5189 (1989).

6. Eyob Alebachew, K. Fesseha. Interaction of a two-level atom with squeezed light. Opt. Commun. 271, 154 (2007).

7. N.A. Ansari, J. Gea-Banacloche, M.S. Zubairy. Phasesensitive amplification in a three-level atomic system. Phys. Rev. A 41, 5179 (1990).

8. N.A. Ansari. Effect of atomic coherence on the second and higher-order squeezing in a two-photon three-level cascade atomic system. Phys. Rev. A 48, 4686 (1993).

9. S.M. Barnett, P.M. Radmore. Methods in Theoretical Quantum Optics (Oxford Univ. Press, 1997).

10. M.O. Scully, K. Wodkiewicz, M.S. Zubairy, J. Bergou, N. Lu, J. Meyer ter Vehn. Two-photon correlated-spontaneous-emission laser: Quantum noise quenching and squeezing. Phys. Rev. Lett. 60, 1832 (1988).

11. C.W. Gardiner, P. Zoller. Quantum Noise (Springer, 2000).

12. D.F. Walls. Squeezed states of light. Nature 306, 141 (1983).

13. A. Abramovici, et al. The laser interferometer gravitational-wave observatory. Science 256, 325 (1992).
14. J. Harms, et al. Squeezed-input, optical-spring, signalrecycled gravitational-wave detectors. Phys. Rev. D 68 042001 (2003).

15. R. Schnabel, et al. Squeezed light and laser interferometric gravitational wave detectors. Classical Quant. Grav. 25 1045 (2004)

Received 06.08.19

М. Молла Гессесс

ДІЯ ШУМУ ВАКУУМНОГО

РЕЗЕРВУАРА НА ДИНАМІКУ ТРИРІВНЕВОГО

ЛАЗЕРА 3 НАКАЧУВАННЯМ

КОГЕРЕНТНИМ СВІТЛОМ

Р е $з$ ю м е

Досліджено дію шуму вакуумного резервуара на статистику фотонів і квадратурне стиснення світла, що генерується трирівневим лазером при накачуванні когерентним світлом і зв'язком з резервуаром за допомогою однопортового дзеркала. Показано, що такий шум збільшує дисперсію числа фотонів і зменшує квадратурне стиснення, не змінюючи середнього числа фотонів. 